\title{
LIMITATIONS ON MEASURING A TRANSVERSE PROFILE OF ULTRA- DENSE ELECTRON BEAMS WITH SCINTILLATORS
}

\author{
A. Murokh*, J. Rosenzweig, University of California, Los Angeles, CA 90095-1547, USA \\ I. Ben-Zvi, X. Wang, V. Yakimenko ${ }^{\dagger}$, Brookhaven National Laboratory, Upton, NY 11973, USA
}

\begin{abstract}
As the high-brightness electron beams become denser and reach sub- $100 \mu \mathrm{m}$ sizes, a resolution of the transverse profile diagnostics become an important issue. The applicability of the traditional scintillators as the diagnostics for most generic transverse properties of small dense beams is questionable. Recently, a YAG:Ce single crystal was used successfully, but studies indicated the presence of saturation effects. At BNL-ATF (Accelerator Test Facility) we have generated ultra-small beams, and continued an experimental work to further illuminate the nature of YAG:Ce saturation. We have also evaluated alternative diagnostics, such as LuAG:Ce. Imaging properties of various diagnostics were studied, including measurements of emission spectra.
\end{abstract}

\section{INTRODUCTION}

Imaging of the electron beams is often critical to the successful operation of the relativistic electron beam sources. Yet the phosphorecent materials most commonly used for this purpose has been know to have resolution limits, traditionally associated with the finite grain structure. In addition, the response time of the scintillators can be as long as microseconds, which can result in a saturation of the emission sites by an excessive amount of the charge deposited.

Most widespread alternative to the scintillators is an imaging of the optical transition radiation (OTR), emitted from a media boundary intercepting the path of the electron beam. OTR in principle allows sub-micron resolution, but the drawback is a low signal intensity, which limits the dynamic range of the diagnostic system to the high brightness electron beams.

Use of a YAG:Ce [1] single crystals appeared to be a promising alternative to the traditional phosphors, by removing the grain size constrain off the resolution limit [2], and in the same time allowing an excellent dynamic range, combined with the vacuum-friendly physical properties. Yet, some recent studies have indicated that the YAG:Ce image of the high brightness beams focused to a tight spot is not adequate to the corresponding OTR image [3]. Last year, in the experiment conducted at ATF we demonstrated that indeed there is a big discrepancy between the beam sizes measured with OTR and wire scan technique, compare to those measured with the scintillators, including YAG:Ce crystals [4]. We have also developed theoretical models which could contribute to

*murokh@physics.ucla.edu

†yakimenko@bnl.gov the effect. In particular, we (1) ruled out a depth of focus problem associated with the crystal finite thickness; (2) examined the scintillation process in YAG:Ce and studied a possibility of saturating the population of the emission sites; and (3) we looked into the collective effects associated with the space charge of the electron beam.

Recently we followed up with another round of the experiment at ATF, where the larger variety of the diagnostic materials had been tested, to specifically examine if the nature and the concentration of scintillating sites will play a major role in the observed "imageblurring" effect. This time a permanent magnets focusing cell was used to achieve a small transverse size of the test beam.

\section{PRODUCING ULTRA-SMALL BEAMS}

The experiment was performed at the Beam line 1 of the Accelerator Test Facility [5]. The electron bunches generated by the photo-injector, were accelerated to 50 $\mathrm{MeV}$ in the linac, and then transported through the dispersion section into the experimental area (see Fig. 1). The optics is tuned to compensate for the dispersion at the entrance of the Beam line 1, yet allowing an energy spread monitoring in the non-zero dispersion region. The beam line was initially tuned by comparing the actual measured beam sizes to the MAD simulations. The first quadrupole triplet on Beam line 1 produces a local beam waist on the downstream beam profile monitor, which generates a controlled and reproducible starting point for

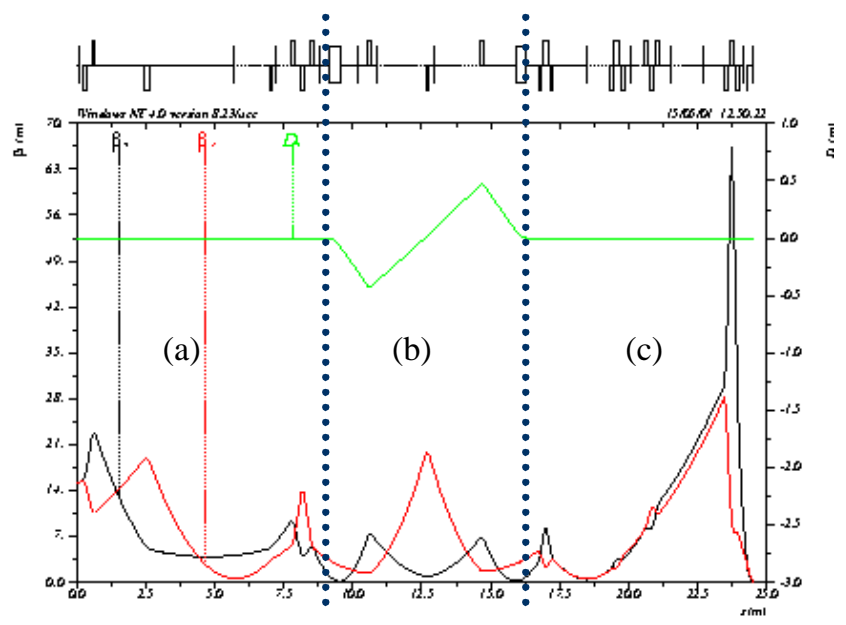

Figure 1: Evolution of the electron beam $\beta$-functions and a dispersion function along (a) post linac focusing channel; (b) dispersion section; (c) beam line 1 focusing optics. 


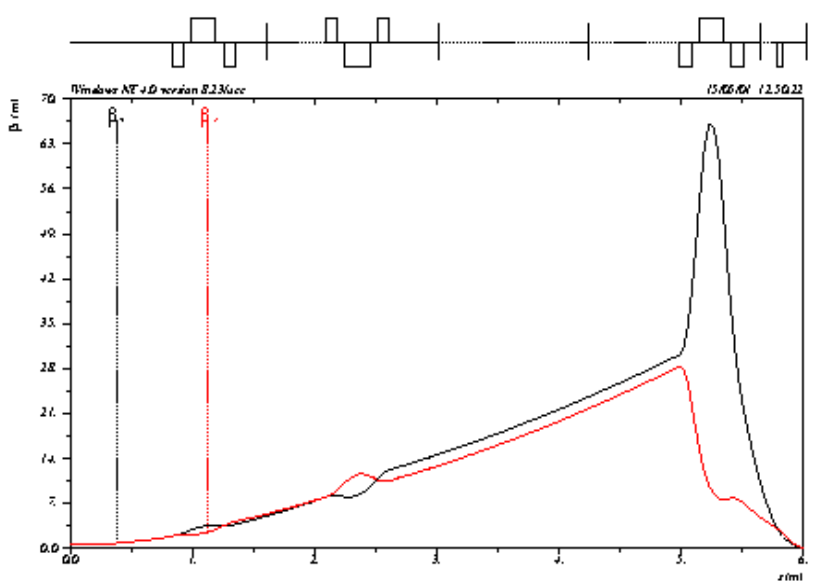

Figure 2: Beam optics layout including a permanent quadrupoles cell at the final focus.

the rest of the beam line.

The final focus is achieved within the last 6 meters of the beam line (Fig. 2). Two regular quadrupole triplets followed by the permanent magnets in-vacuum assembly form the overall demagnification ratio of 5:1. Betatron phase advance through the whole 6-m section was optimized to be exactly 180 degrees in the both planes. The permanent magnet was located only $20 \mathrm{~cm}$ away from the diagnostics target, which dramatically decreased the chromaticity of the final focus and allowed to generate a 2 -cm $\beta$-function beam in both directions.

\section{DIAGNOSTICS SET-UP}

All the diagnostic samples for the experiment were fixed to the single aluminum bracket, attached to the remotely controlled translation stage. The bracket was positioned at the focus of the in-vacuum parabolic mirror, which enabled to achieve a proper magnification for the optical imaging system. The image resolution on the CCD camera was achieved as small as $3 \mu \mathrm{m} /$ pixel. The optical system acceptance angle was minimized by the motorized iris, so that the depth of field would be

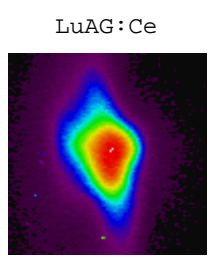

$\mathrm{Gd}_{2} \mathrm{SO}_{3}: \mathrm{Tb}$
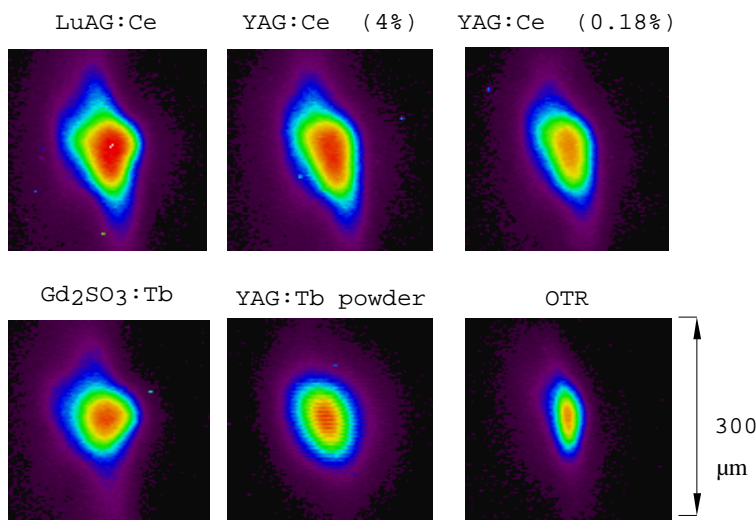

Figure 3: Beam images taken consequently with the six different diagnostics under the stable experimental conditions (the charge $\mathrm{Q} \sim 500 \mathrm{pC}$ ).

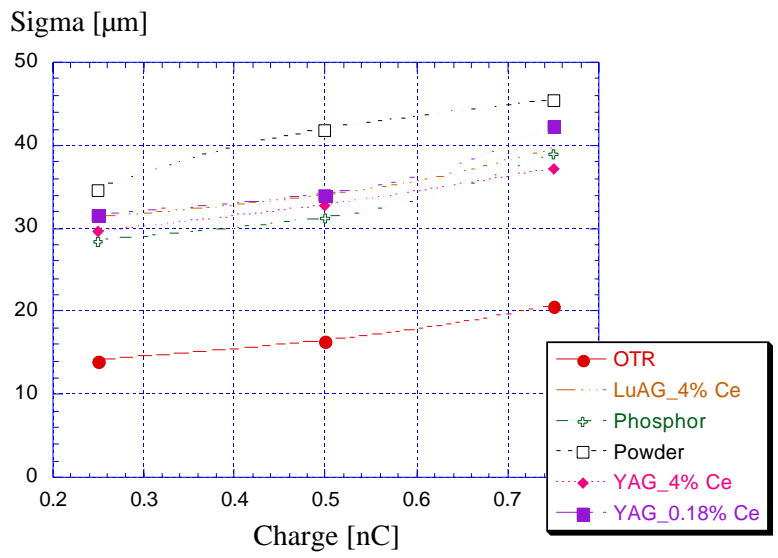

Figure 4: Electron beam horizontal spot size as a function of charge, measured with the scintillating diagnostics and the OTR.

sufficient to preserve the high resolution within $0.2 \mathrm{~mm}$ thick crystals. Operating the translation stage allowed multiplexing between the diagnostic samples without changing any properties of neither the electron beam nor the imaging system. The set of 6 diagnostics samples has been tested:

- $\quad$ YAG single crystal doped with $0.18 \% \mathrm{Ce}$, used in previous experiments.

- $\quad$ YAG single crystal doped with $4.0 \% \mathrm{Ce}$.

- $\quad$ LuAG single crystal doped with $4.0 \%$ Ce. Chemical and physical properties of this crystal are identical to the ones of the YAG, yet larger band gap $(12 \mathrm{MeV})$ makes the ion yield in the crystal less efficient; hence, making the material more resistant to the depletion of the emission sites by the beam of an excessive density.

- YAG:Tb powder screen with the ultra-small grain structure.

- Terbium doped gadolinium sulfa-oxide screen (ATF homemade phosphor which is commonly used at the accelerator labs for many years)

- $\quad \lambda / 2$ aluminized mirror for the OTR measurements.

\section{RESULTS AND DISCUSSION}

At the beginning of the run the beam spot size was minimized by varying the quadrupole settings. It is noteworthy, that in this process the strengths of the last two quadrupoles were significantly adjusted compare to the original simulated values. That can be explained by a significance of the space charge term in this configuration. Since there were no sextupoles installed to compensate for the chromaticity of the final focus state, it was not surprising, that the jitter in the linac resulted in the observable increase of the beam size on the target. Fortunately, the size fluctuations were cyclical and relatively small; therefore they did not affect any of the diagnostic measurements.

Initial comparison of the beam sizes as measured on the different diagnostics (Fig. 3) agreed with our previous 

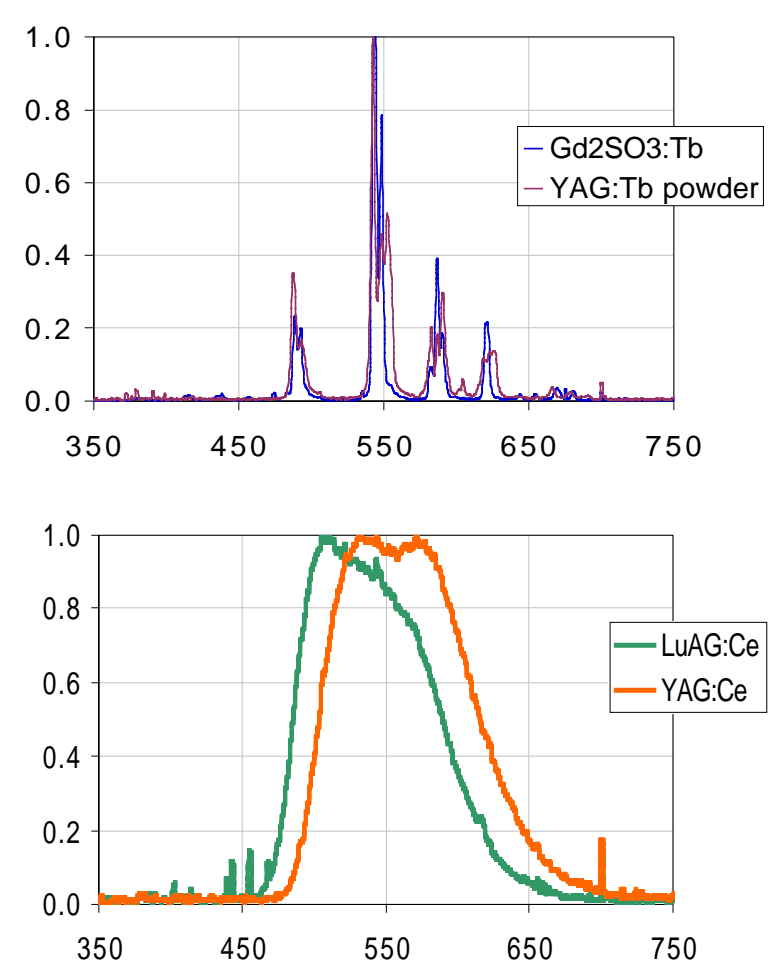

Figure 5: Spectral profiles of $\mathrm{Tb}$ doped and $\mathrm{Ce}$ scintillators emission respectively, under the electron beam excitation. Small dip at $550 \mathrm{~nm}$ in the YAG:Ce emission profile was very distinct and repeatable.

observations [4]. All the scintillators images are significantly larger than the OTR image.

One can notice only the minor differences in scintillators performance (with the exception of YAG:Tb powder) as the measurements were repeated for the different charges (Fig. 4). Both YAG and LuAG crystals with $4 \%$ Ce doping, showed smoothly increasing image size closely resembling the OTR measurements; scaled, however, by almost a factor of 2 . In addition the images aspect ratios (Fig. 3) were found similar to the OTR image.

The performances of the standard YAG:Ce crystal with $0.18 \%$ dopant concentrationand the regular phosphor screen $\left(\mathrm{Gd}_{2} \mathrm{SO}_{3}: \mathrm{Tb}\right)$ are very similar to the ones of $4 \%$ crystals at the smaller beam charges. As the charge increases, though, the measured beam sizes start growing at a faster rate, and beam images aspect ratio starts deviating significantly from the OTR data. These observations may indicate the emission sites depletion, as the electron beam cross-section approaches the limit, where in the process of the impact, a density of electronhole pairs produced [4] exceeds a local concentration of the Ce ions available for the emission process.

The only outstanding scintillating diagnostic was a YAG:Tb powder sample. The corresponding image of the beam completely lacks the characteristic shape (Fig. 3), and shows even larger size than the rest of the scintillators. One explanation could be the fact that the powder grain size is very small which causes most of the electron-hole pairs to be trapped at the boundaries and decay through the non-radiative quenching in the $\mathrm{Ce}$ deficient environment [6].

Overall we can conclude, that the difference in sizes of the beam images generated with the OTR and the scintillators emission is very distinct, and within the limits is rather independent of the scintillators properties, such as dopant concentration, band gap size, and the crystalline structure. That indicates, that the nature of the observed "image-blurring" is rather generic and may be related to the collective effects exhibited by the electron beam itself. Example of such an effect was discussed in our previous work [4], where we hypothesized, that the space charge of the beam creates an additional ionization cascades, seeded by the original scattering ionization. That will multiply ion pairs density in accordance with the space charge field profile which is generally larger than the beam itself.

In addition to the direct image measurements, we used the similar set-up to look at the emission spectra of the scintillated materials (Fig. 5). The generic spectral properties of the studied materials are well characterized through the optical and $\gamma$ excitations [7]; yet, we decided to investigate the spectral properties of the studied materials, under the high brightness electron beam excitation, searching for some indications of collective behavior. Generally the measurements are in the good agreement with $\gamma$ excitations studies [7]. One unusual feature, though, is a very repeatable dip at the $550 \mathrm{~nm}$ point of YAG:Ce emission line, just were the sharp peak is expected. It may indicate some form of the light reabsorption, but at the moment we can not offer any satisfactory model to explain this observation.

\section{ACKNOWLEDGEMENT}

The authors would like to thank Bob Harrington, Karl Kusche, Igor Pogorelsky and Frank Stephan, for their help and support. This work was supported by the DOE grants DE-AC02-98CH10886 and DE-FG03-92ER40693.

\section{REFERENCES}

[1] http://www.crytur.cz/

[2] W.S. Graves et al., PAC 97 Proceedings, 1993-1995 (1998)

[3] A.H. Lumpkin et al., Nucl. Instr. and Meth. A 429, 336-340 (1999)

[4] A. Murokh et al., Proceedings of the $2^{\text {nd }}$ ICFA Advanced Accelerator Workshop, 564-580 (2000)

[5] X. Wang et al., PAC 99 Proceedings, 3495 (1999) [6]

[6] E. Zych et al., Journal of Luminescence 75, 193-203 (1997)

[7] E. Lempicki et al., IEEE Transactions on Nuclear Science 42(4), 280-284 (1995) 